\title{
Recent Advances in Efficient Computation of Deep Convolutional Neural Networks
}

\author{
Jian Cheng, Peisong Wang, Gang Li, Qinghao Hu, Hanqing Lu \\ National Laboratory of Pattern Recognition, Institute of Automation, Chinese Academy of Sciences \\ University of Chinese Academy of Sciences \\ jcheng@nlpr.ia.ac.cn
}

\begin{abstract}
Deep neural networks have evolved remarkably over the past few years and they are currently the fundamental tools of many intelligent systems. At the same time, the computational complexity and resource consumption of these networks also continue to increase. This will pose a significant challenge to the deployment of such networks, especially in real-time applications or on resource-limited devices. Thus, network acceleration has become a hot topic within the deep learning community. As for hardware implementation of deep neural networks, a batch of accelerators based on FPGA/ASIC have been proposed in recent years. In this paper, we provide a comprehensive survey of recent advances in network acceleration, compression and accelerator design from both algorithm and hardware points of view. Specifically, we provide a thorough analysis of each of the following topics: network pruning, low-rank approximation, network quantization, teacher-student networks, compact network design and hardware accelerators. Finally, we will introduce and discuss a few possible future directions.
\end{abstract}

\section{Introduction}

In recent years, deep neural networks (DNNs) have achieved remarkable performance across a wide range of applications, including but not limited to computer vision, natural language processing, speech recognition, etc. These breakthroughs are closely related to the increased amount of training data and more powerful computing resources now available. For example, one breakthrough in the natural image recognition field was achieved by AlexNet [44], which was trained using multiple graphics processing units (GPUs) on about 1.2M images. Since then, the performance of DNNs has continued to improve. For many tasks, DNNs are reported to be able to outperform humans. The problem,

\footnotetext{
${ }^{*}$ The corresponding author.
}

however, is that the computational complexity as well as the storage requirements of these DNNs has also increased drastically as shown in Table 1 Specifically, the widely used VGG-16 model [72] involves more than 500MB of storage and over 15B FLOPs to classify a single $224 \times 224$ image.

Thanks to the recent crop of powerful GPUs and CPU clusters equipped with more abundant memory resources and computational units, these more powerful DNNs can be trained within a relatively reasonable time period. However, when it is time for the inference phase, such a long execution time is impractical for real-time applications. Recent years have witnessed great progress in embedded and mobile devices including unmanned drones, smart phones, intelligent glasses, etc. The demand for deployment of DNN models on these devices has become more intense. However, the resources of these devices, for example, the storage and computational units as well as the battery power remain very limited, and this poses a real challenge in accelerating modern DNNs in low-cost settings.

Therefore, a critical problem currently is how to equip specific hardware with efficient deep networks without significantly lowering the performance. To deal with this issue, many great ideas and methods from the algorithm side have been investigated over the past few years. Some of these works focused on model compression while others focused on acceleration or lowering power consumption. As for the hardware side, a wide variety of FPGA/ASIC-based accelerators have been proposed for embedded and mobile applications. In this paper, we present a comprehensive survey of several advanced approaches in network compression, acceleration and accelerator design. We will present the central ideas behind each approach and explore the similarities and differences between the different methods. Finally, we will present some future directions in the field.

The rest of this paper is organized as follows. In Section 2, we give some background on network acceleration and compression. From Section 3 to Section 7, we systematically describe a series of hardware-efficient DNN al- 
gorithms, including network pruning, low-rank approximation, network quantization, teacher-student networks and compact network design. In Section 8, we introduce the design and implementation of hardware accelerators based on FPGA/ASIC technologies. In Section 9, we discuss some future directions in the field, and Section 10 concludes the paper.

\section{Background}

Recently, deep convolutional neural networks (CNNs) have become quite popular due to their powerful representational capacity. With the huge success of CNNs, the demand for deployment of deep networks in real world applications has continued to increase. However, the large storage consumption and computational complexity remain two key problems for deployment of these networks. For the CNN training phase, the computational complexity is not a critical problem thanks to the high performance GPUs or CPU clouds. The large storage consumption also has less effect on the training phase because modern computers have very large disk and memory storage capacities. However, things are quite different for the inference phase in CNNs, especially with regard to embedded and mobile devices.

The enormous computational complexity introduces two problems in the deployment of CNNs in real-world applications. One is that the CNN inference phase slows down as the computational complexity grows larger. This makes it difficult to deploy CNNs in real-time applications. The other problem is that the dense computation inherent to CNNs will consume substantial battery power, which is limited on mobile devices.

The large number of parameters of CNNs consumes considerable storage and run-time memory, which are quite limited on embedded devices. In addition, it becomes more difficult to download new models online on mobile devices.

To solve these problems, network compression and acceleration methods have been proposed. In general, the computational complexity of CNNs is dominated by the convolutional layers, while the number of parameters is mainly related to the fully connected layers as shown in Table 11. Thus, most network acceleration methods focus on decreasing the computational complexity of the convolutional layers, while the network compression methods mainly try to compress the fully connected layers.

\section{Network Pruning}

Pruning methods were proposed before deep learning became popular, and they have been widely studied in recent years [47, 24, 22, 23]. Based on the assumption that many parameters in deep networks are unimportant or unnecessary, pruning methods are used to remove the unimportant parameters. In this way, pruning methods can expand the sparsity of the parameters significantly. The high sparsity of the parameters after pruning introduces two benefits for deep neural networks. On the one hand, the sparse parameters after pruning require less disk storage since the parameters can be stored in the compressed sparse row format (CSR) or compressed sparse column (CSC) format. On the other hand, computations involving those pruned parameters are omitted; thus, the computational complexity of deep networks can be reduced. According to the granularity of the pruning, pruning methods can be categorized into five groups: fine-grained pruning, vector-level pruning, kernellevel pruning, group-level pruning and filter-level pruning. Figure. 1 shows the pruning methods with their different granularities. In the following subsections, we describe the different pruning methods in detail.

\subsection{Fine-grained Pruning}

Fine-grained pruning methods or vanilla pruning methods remove parameters in an unstructured way, i.e., any unimportant parameters in the convolutional kernels can be pruned, as shown in Figure. 1 Since there are no extra constraints on the pruning patterns, the parameters can be pruned with a high sparsity. Early works on pruning [47, 24] used the approximate second-order derivativeses of the loss function w.r.t. the parameters to determine the saliency of the parameters, and then pruned those parameters with low saliency. Yet, deep networks can ill afford to compute the second order derivativeses due to the huge computational complexity. Recently [22] proposed a deep compression framework to compress deep neural networks in three steps: pruning, quantization, and Huffman encoding. By using this method, AlexNet could be compressed by $35 \times$ without drops in accuracy. After pruning, the pruned parameters in [22] remain unchanged, incorrectly pruned parameters could cause accuracy drops. To solve this problem, [17] proposed a dynamic network surgery framework, which consists of two operations: pruning and splicing. The pruning operation aims to prune those unimportant parameters while the splicing operation aims to recover the incorrectly pruned connections. Their method requires fewer training epochs and achieves a better compression ratio than [22].

\subsection{Vector-level and Kernel-level Pruning}

Vector-level pruning methods prune vectors in the convolutional kernels, and kernel-level pruning methods prune 2-D convolutional kernels in the filters. Since most pruning methods focus on fine-grained pruning or filter-level pruning, there are few works on vector-level and kernel-level pruning. [3] first explored the kernel-level pruning, and then proposed an intra-kernel strided pruning method, which prunes a sub-vector in a fixed stride. [58] explored different granularity levels in pruning, and found that vector-level 
Table 1. The computation and parameters for state-of-art convolution neural networks

\begin{tabular}{|c|c|c|c|c|c|c|}
\hline \multirow{2}{*}{ Method } & \multicolumn{3}{|c|}{ Parameters } & \multicolumn{3}{c|}{ Computation } \\
\cline { 2 - 7 } & Size(M) & Conv(\%) & Fc(\%) & FLOPS(G) & Conv(\%) & Fc(\%) \\
\hline AlexNet & 61 & 3.8 & 96.2 & 0.72 & 91.9 & 8.1 \\
\hline VGG-S & 103 & 6.3 & 93.7 & 2.6 & 96.3 & 3.7 \\
\hline VGG16 & 138 & 10.6 & 89.4 & 15.5 & 99.2 & 0.8 \\
\hline NIN & 7.6 & 100 & 0 & 1.1 & 100 & 0 \\
\hline GoogLeNet & 6.9 & 85.1 & 14.9 & 1.6 & 99.9 & 0.1 \\
\hline ResNet-18 & 5.6 & 100 & 0 & 1.8 & 100 & 0 \\
\hline ResNet-50 & 12.2 & 100 & 0 & 3.8 & 100 & 0 \\
\hline ResNet-101 & 21.2 & 100 & 0 & 7.6 & 100 & 0 \\
\hline
\end{tabular}
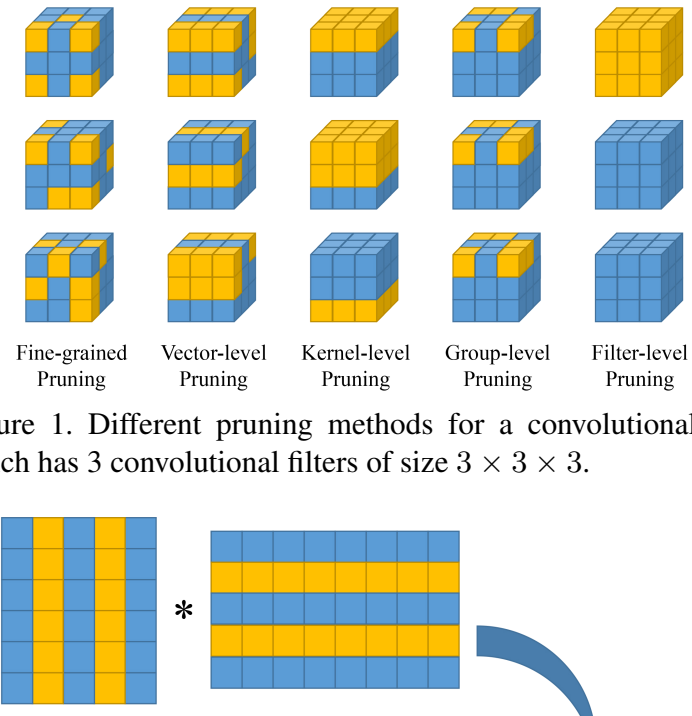

Vector-leve
Pruning

Kernel-level

Group-level
Pruning

Filter-level
Pruning

Pruning

Figure 1. Different pruning methods for a convolutional layer which has 3 convolutional filters of size $3 \times 3 \times 3$.

Weight Matrix

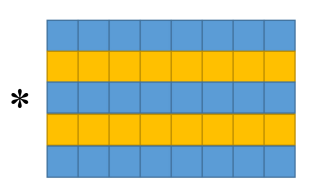

Featuremap Patches
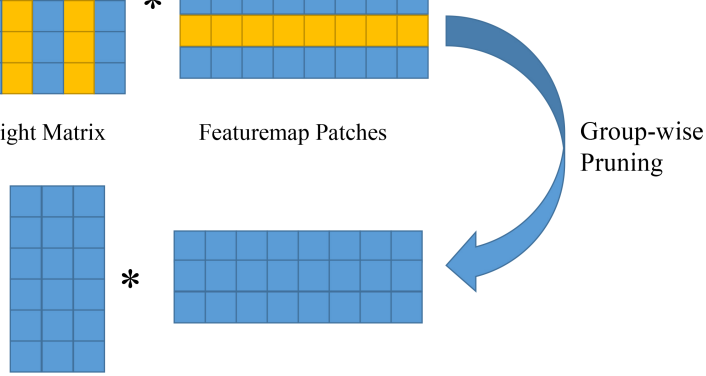

Thin Weight Matrix Reduced Featuremap Patches

Figure 2. Group-level Pruning.

pruning takes up less storage than fine-grained pruning because vector-level pruning requires fewer indices to indicate the pruned parameters. Nevertheless, vector-level, kernellevel, and filter-level pruning techniques are friendlier in hardware implementations since they are the more structured pruning methods.

\subsection{Group-level Pruning}

Group-level pruning methods prune the parameters according to the same sparse pattern on the filters. As shown in Figure 2, each filter has the same sparsity pattern, thus the convolutional filters can be represented as a thinned dense matrix. By using group-level pruning, convolutions can be implemented by thinned dense matrices multiplication. As a result, the Basic Linear Algebra Subprograms (BLAS) can be utilized to achieve a higher speed-up. [46] proposed the group-wise brain damage approach, which prunes the weight matrix in a group-wise fashion. By using groupsparsity regularization, deep networks can be trained easily with group-sparsified parameters. Since group-level pruning can utilize the BLAS library, the practical speed-up is almost linear at the sparsity level. By using this method, they achieved a $3.2 \times$ speed-up for all convolutional layers in AlexNet. Concurrent with [46], [85] proposed using the group Lasso to prune groups of parameters. In contrast, [85] explored different levels of structured sparsity in terms of filters, channels, filter shapes, and depth. Their method can be regarded as a more general group-regularized pruning method. For AlexNet's convolutional layers, their method achieves about $5.1 \times$ and $3.1 \times$ speed-ups on a CPU and GPU respectively.

\subsection{Filter-level Pruning}

Filter-level pruning methods prune the convolutional filters or channels which make the deep networks thinner. After the filter pruning for one layer, the number of input channels of the next layer is also reduced. Thus, filter-level pruning is more efficient for accelerating deep networks. [54] proposed a filter-level pruning method named ThiNet. They used the next layer's feature map to guide the filter pruning in the current layer. By minimizing the feature map's reconstruction errors, they select the channels in a greedy way. Similar to [54], [26] proposed an iterative two-step algorithm to prune filters by minimizing the feature map errors. Specifically, they introduced a selection weight $\beta_{i}$ for each filter $\boldsymbol{W}_{\boldsymbol{i}}$, then added sparse constraints on $\beta_{i}$. Then the channel selection problem can be casted into a LASSO regression problem. To minimize the feature map errors, they iteratively updated $\beta$ and $\boldsymbol{W}$. Moreover, their method achieved a $5 \times$ speed-up on VGG-16 network with little drop in accuracy. Instead of using additional selection weight $\beta$, [53] proposed to leverage the scaling factor of the batch normalization layer for to evaluate the importance of 
the filters. By pruning the channels with near-zero scaling factors, they can prune filters without introducing overhead into the networks.

\section{Low-rank Approximation}

The convolutional kernel of a convolutional layer $\boldsymbol{W} \in$ $R^{w \times h \times c \times n}$ is a 4-D tensor. These four dimensions correspond to the kernel width, kernel height and the number of input and output channels respectively. Note that by merging some of the dimensions, the 4-D tensor can be transformed into a $t$-D $(t=1, \cdots 4)$ tensor. The motivation behind low-rank decomposition is to find an approximate tensor $\hat{\boldsymbol{W}}$ that is close to $\boldsymbol{W}$ but facilitates more efficient computation. Many low-rank based methods have been proposed by the community; two key differences are in how to rearrange the four dimensions, and on which dimension the low-rank constraint is imposed. Here we roughly divide the low-rank based methods into three categories, according to how many components the filters are decomposed into: two-component decomposition, three-component decomposition and four-component decomposition.

\subsection{Two-component Decomposition}

For two-component decomposition, the weight tensor is divided into two parts and the convolutional layer is replaced by two successive layers. [35] decomposed the spatial dimension $w * h$ into $w * 1$ and $1 * h$ filters. They achieved a $4.5 \times$ speedup for a $\mathrm{CNN}$ trained on a text character recognition dataset, with a $1 \%$ accuracy drop.

SVD is a popular low-rank matrix decomposition method. By merging the dimensions $w, h$ and $c$, the kernel becomes a 2-D matrix of size $(w * h * c) \times n$, on which the SVD decomposition method can be conducted. In [11], the authors utilized SVD to reduce the network redundancy. SVD decomposition was also investigated in [97], in which the filters were replaced by two filter banks: one consisting of $d$ filters of shape $w \times h \times c$ and the other composed of $n$ filters of shape $1 \times 1 \times d$. Here, $d$ represents the rank of the decomposition, i.e., the $n$ filters are linear combinations of the first $d$ filters. They also proposed the non-linear response reconstruction method based on the low-rank decomposition. On the challenging VGG-16 model for the ImageNet classification task, this two-component SVD decomposition method achieved a $3 \times$ theoretical speedup at a cost of about $1.66 \%$ increased top-5 error.

Similarly, another SVD decomposition method can be used by exploring the low-rank property along the input channel dimension $c$. In this way, we reshape the weight tensor into a matrix of size $c \times(w * h * n)$. By selecting the rank to $d$, the convolution can be decomposed first by a $1 \times 1 \times c \times d$ convolution and then by a $w \times h \times d \times n$ convolution. These two decomposition are symmetric.

\subsection{Three-component Decomposition}

Based on the analysis of two-component decomposition methods, one straightforward three-component decomposition method can be obtained by two successive twocomponent decompositions. Note that in the SVD decomposition, two weight tensors are introduced. The first is a $w \times h \times c \times d$ tensor and the other is a $d \times n$ tensor (matrix). The first convolution is also very time consuming due to the large size of the first tensor. We can also conduct a two-component decomposition on the first weight tensor after the SVD decomposition, which turns into a threecomponent decomposition method. This strategy was studied by [97], whereby after the SVD decomposition, they utilized the decomposition method proposed by [35] for the first decomposed tensor. Thus, the final three components were convolutions with a spatial size of $w \times 1,1 \times h$, and $1 \times 1$, respectively. By utilizing this three-component decomposition, only a $0.3 \%$ increased top- 5 error was produced in [97] for a $4 \times$ theoretical speedup.

If we use the SVD decomposition along the input channel dimension for the first tensor after the two-component decomposition, we can get the Tucker decomposition format as proposed by [41]. These three components are convolutions of a spatial size $1 \times 1, w \times h$ and another $1 \times 1$ convolution. Note that instead of using the twostep SVD decomposition, [41] utilized the Tucker decomposition method directly to obtain these three components. Their method achieved a $4.93 \times$ theoretical speedup at a cost of $0.5 \%$ increased top- 5 accuracy.

To further reduce complexity, [80] proposed a BlockTerm Decomposition (BTD) method based on low-rank and group sparse decomposition. Note that in the Tucker decomposition, the second component corresponding to the $w \times h$ convolution also requires a large number of computations. Because the second tensor is already low rank along both the input and output channel dimensions, the decomposition methods discussed above cannot be used any longer. [80] proposed to approximate the original weight tensor by the sum of some smaller subtensors, each of which is in the Tucker decomposition format. By rearranging these subtensors, the BTD can be seen as a Tucker decomposition where the second decomposed tensor is a block diagonal tensor. By using this decomposition, they achieved a $7.4 \%$ actual speedup for the VGG-16 model, at a cost of a 1.3\% increased in the top-5 error. Their method also achieved high speedup for object detection and image retrieval tasks as reported in [82].

\subsection{Four-component Decomposition}

By exploring the low-rank property along the input/output channel dimension as well as the spatial dimension, a four-component decomposition can be obtained. This is corresponds to the CP-decomposition acceleration 
method proposed in [45]. In this way, the four components are convolutions of size $1 \times 1, w \times 1,1 \times h$ and $1 \times 1$. The CP-decomposition can achieve a very high speedup ratio, however, due to the approximate error, only the second layer of AlexNet was processed in [45]. They achieved a $4.5 \times$ speedup for the second layer of AlexNet at a cost of about a $1 \%$ accuracy drop.

\section{Network Quantization}

Quantization is an approach for many compression and acceleration applications. It has wide applications in image compression, information retrieval, etc. Many quantization methods have also been investigated for network acceleration and compression. We can categorize these methods into two main groups: (1) scalar and vector quantization, which may need a codebook for quantization, and (2) fixedpoint quantization.

\subsection{Scalar and Vector Quantization}

Scalar and vector quantization techniques have a long history, and they were originally used for data compression. By using scalar or vector quantization, the original data can be represented by a codebook and a set of quantization codes. The codebook contains a set of quantization centers, and the quantization codes are used to indicate the assignment of the quantization centers. In general, the number of quantization centers is far smaller than the amount of original data. In addition, quantization codes can be encoded through a lossless encoding method, e.g., Huffman coding, or just represented as low-bit fixed points. Thus, scalar or vector quantization can achieve a high compression ratio. [15] explored scalar and vector quantization techniques for compressing deep networks. For scalar quantization, they used the well-known $K$-means algorithm to compress the parameters. In addition, the product quantization algorithm (PQ) [36], a special case of vector quantization, was leveraged to compress the fully connected layers. By partitioning the feature space into several disjoint subspaces and then conducting $K$-means in each subspace, the PQ algorithm can compress the fully connected layers with little loss. As [15] only compressed the fully connected layers, in [86] and [8], the authors proposed to utilize the PQ algorithm to simultaneously accelerate and compress convolutional neural networks. They proposed to quantize the convolutional filters layer by layer by minimizing the feature map's reconstruction loss. During the inference phase, a look-up table is built by pre-computing the inner product between feature map patches and codebooks, then the output feature map can be calculated by simply accessing the look-up table. By using this method, they can achieve $4 \sim 6 \times$ speedup and 15 $\sim 20 \times$ compression ratio with little accuracy loss.

\subsection{Fixed-point Quantization}

Fixed-point quantization is an effective approach for lowering the resource consumption of a network. Based on which part is quantized, two main categories can be classified, i.e., weight quantization and activation quantization. There are some other works that try to also quantize gradients, which can result in acceleration at the network training stage. Here, we mainly review weight quantization and activation quantization methods, which accelerate the testphase computation. Table 2 summarizes these methods according to which part is quantized and whether the training and testing stages can be accelerated.

\subsubsection{Fixed-point Quantization of Weights}

Fixed-point weight quantization is a fairly mature topic in network acceleration and compression. [19] proposed a VLSI architecture for network acceleration using 8-bit input and output, and 16-bit internal representation. In [28], the authors provided a theoretical analysis of error caused by low-bit quantization to determine the bitwidth for a multilayer perceptron. They showed that $8 \sim 16$ bit quantization was sufficient for training small neural networks. These early works mainly focused on simple multilayer perceptrons. A more recent work [6] showed that it is necessary to use 32-bit fixed-point for the convergence of a convolutional neural network trained on MNIST dataset. By using stochastic rounding, the work by [18] found that it is sufficient to use 16-bit fixed-point numbers to train a convolutional neural network on MNIST. In addition, 8-bit fixedpoint quantization was also investigated in [12] to speed up the convergence of deep networks in parallel training. Logarithmic data representation was also investigated in [59].

Recently, much lower bit quantization or even binary and ternary quantization methods have been investigated. Expectation Backpropagation (EBP) was introduced in [9], which utilized the variational Bayes method to binarize the network. The BinaryConnect method proposed in [10] constrained all weights to be either +1 or -1 . By training from scratch, the BinaryConnect can even outperform the floating-point counterpart on the CIFAR-10 [43] image classification dataset. Using binary quantization, the network can be compressed about 32 times compared to 32-bit floating-point networks. Most of the floating-point multiplication can also be eliminated [51]. In [65], the authors proposed the Binary Weight Network (BWN), which was among the earliest works that achieved good results on the large ImageNet [68] dataset. Loss-aware binarization was proposed in $([30])$, which can directly minimize the classification loss with respect to the binarized weights. In the work of [32], the authors proposed a novel approach called BWNH to train Binary Weight Networks via Hashing, which outperformed other weight binarization methods 
Table 2. Comparison of fixed-point quantization methods according to which part is quantized and whether the training and testing stages can be accelerated.

\begin{tabular}{|c|c|c|c|c|c|}
\hline \multirow{2}{*}{ Method } & \multicolumn{3}{|c|}{ Quantization } & \multicolumn{2}{c|}{ Acceleration } \\
\cline { 2 - 6 } & Weight & Activation & Gradient & Training & Testing \\
\hline BinaryConnect [10] & Binary & Full & Full & No & Yes \\
\hline BWN [65] & Binary & Full & Full & No & Yes \\
\hline BWNH [32] & Binary & Full & Full & No & Yes \\
\hline TWN [48] & Binary & Full & Full & No & Yes \\
\hline FFN [81] & Ternary & Full & Full & No & Yes \\
\hline INQ [99] & Ternary-5bit & Full & Full & No & Yes \\
\hline BNN [65] & Binary & Binary & Full & No & Yes \\
\hline XNOR [65] & Binary & Binary & Full & No & Yes \\
\hline HWGQ [4] & Binary & 2bit & Full & No & Yes \\
\hline DoReFa-Net [100] & Binary & $1-4$ bit & 6bit, 8bit, Full & Yes & Yes \\
\hline
\end{tabular}

by a large margin. Ternary quantization was also utilized in [33]. In [48], the authors proposed the Ternary Weight Network (TWN), which was similar to BWN, but constrained all weights to be ternary values among $\{-1,0,+1\}$. The TWN outperformed BWN by a large margin on deep models like ResNet. Trained Ternary Quantization proposed in [101] learned both ternary values and ternary assignments at the same time using back-propagation. They achieved comparable results on the AlexNet model. Different from previous quantization methods, the Incremental Network Quantization (INQ) method proposed in [99] gradually turned all weights into a logarithmic format in a multi-step manner. This incremental quantization strategy can lower the quantization error during each stage, and thus can make the quantization problem much easier. All these low-bit quantization methods discussed above directly quantize the full-precision weight into a fixed-point format. In [81], the authors proposed a very different quantization strategy. In stead of direct quantization, they proposed using a fixedpoint factorized network (FFN) to quantize all weights into ternary values. This fixed-point decomposition method can significantly lower the quantization error. The FFN method achieved comparable results on commonly used deep models such as AlexNet, VGG-16 and ResNet.

\subsubsection{Fixed-point Quantization of Activations}

Given only weight quantization, there is also a need for the time-consuming floating-point operations. If the activations were also quantized into fixed-point values, the network can be efficiently executed by only fixed-point operations. Many activation quantization methods were also proposed by the deep learning community. The bitwise neural network was proposed in [40]. Binarized Neural Networks (BNN) were among the first works that quantized both weights and activations into either -1 or +1 . BNN achieved a comparable accuracy with the full-precision baseline on the CIFAR-10 dataset. To extend the BNN for the ImageNet classification task, the authors in [75] improved the training strategies of the BNN. Much higher accuracy was reported using these strategies. Based on the BWN, the authors in [65] further quantize all activations into binary values, making the network into a XNOR-Net. Compared with BNN, the XNOR-Net can achieve much higher accuracy on the ImageNet dataset. To further understand the effect of bit-width on the training of deep neural networks, the DoReFa-Net was proposed in [100]. It investigated the effect of different bit-widths for weights and activations as well as gradients. By making use of batch normalization, the work by [4] presented the Half-wave Gaussian Quantization (HWGQ) method to quantize both weights and activations. A high performance was achieved on commonly used CNN models using the HWGQ methond, with 2-bit activations and binary weights.

\section{Teacher-student Network}

The teacher-student network is different from the network compression or acceleration methods since it trains a student network using a teacher network and the student network can be designed with a different network architecture. Generally speaking, a teacher network is a large neural network or the ensemble of neural networks while a student network is a compact and efficient neural network. By utilizing the dark knowledge transferred from the teacher network, the student network can achieve higher accuracy than training merely through the class labels. [27] proposed the knowledge distillation (KD) method which trains a student network by the softmax layer's output of the teacher network. Following this line of thinking, [67] proposed the FitNets to train a deeper and thinner student network. Since the depth of neural networks is more important than the width of them, a deeper student network would have higher accuracy. Besides, they utilized both intermediate layers' featuremaps and soft outputs of the teacher network to train the student network. Rather than mimicking the intermediate layers' feature maps, [91] proposed to train a student network by imitating the attention maps of a teacher network. 
Their experiments showed that the attention maps are more important than the layers' activations and their method can achieve higher accuracy than FitNets.

\section{Compact Network Design}

The objective of network acceleration and compression is to optimize the execution and storage framework for a given deep neural network. One property is that the network architecture is not changed. Another parallel line of inquiry for network acceleration and compression is to design more efficient but low-cost network architecture itself.

In [50], the authors proposed Network-In-Network architecture, where a $1 \times 1$ convolution was utilized to increase the network capacity while keeping the overall computational complexity small. To reduce the storage requirement of the CNN models, they also proposed to remove the fully connected layer and make use of a global average pooling. These strategies are also used by many state-of-the-art CNN models like GoogLeNet [74] and ResNet [25].

Branching (multiple group convolution) is another commonly used strategy for lowering network complexity, which was explored in the work of GoogLeNet [74]. By largely making use of $1 \times 1$ convolution and the branching strategy, the SqueezeNet proposed in [34] achieved about $50 \times$ compression over AlexNet, with comparable accuracy. By branching, the work of ResNeXt work of [89] can achieve much higher accuracy than the ResNet [25] at the same computational budget. The depth-wise convolution proposed in MobileNet [31] takes the branching strategy to the extreme, i.e., the number of branches equals the number of input/output channels. The resulting MobileNet can be $32 \times$ smaller and $27 \times$ faster than the VGG16 model, with comparable image classification accuracy on ImageNet. When using depth-wise convolution and $1 \times 1$ convolution as in MobileNet, most of the computation and parameters reside in the $1 \times 1$ convolutions. One strategy to further lower the complexity of the $1 \times 1$ convolution is to use multiple groups. The ShuffleNet proposed in [96] introduced the channel shuffle operation to increase the information change within the multiple groups, which can prominently increase the representational power of the networks. Their method achieved about $13 \times$ actual speedup over AlexNet with comparable accuracy.

\section{Hardware Accelerator}

\subsection{Background}

Deep neural networks provide impressive performance for various tasks while suffering from degrees of computational complexity. Traditionally, algorithms based on deep neural networks should be executed on general purpose platforms such as CPUs and GPUs, but this works at the expense of unexpected power consumption and oversized

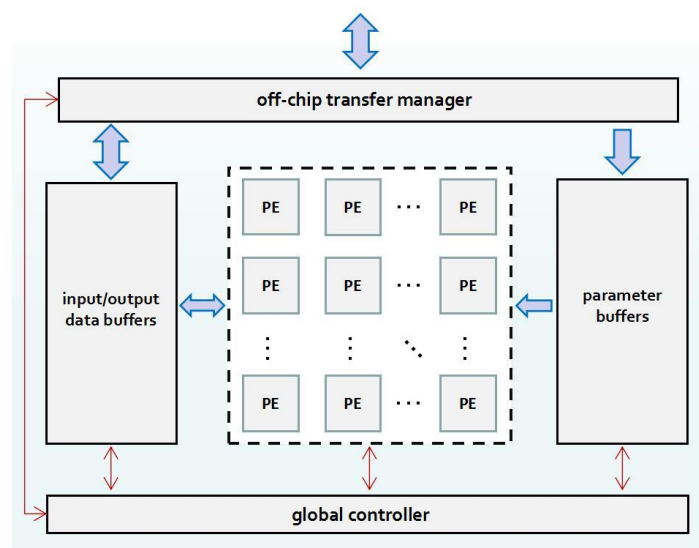

Figure 3. General architecture of an accelerator on dedicated hardware.

resource utilization for both computing and storage. In recent years, there are an increasing number of applications that are based on embedded systems, including autonomous vehicles, unmanned drones, security cameras, etc. Considering the demands for high performance, light weight and low power consumption on these devices, CPU/GPUbased solutions are no longer suitable. In this scenario, FPGA/ASIC-based hardware accelerators are gaining popularity as efficient alternatives.

\subsection{General Architecture}

The deployment of a DNN on a real-world application consists of two phases: training and inference. Network training is known to be expensive in terms of speed and memory, thus it is usually carried out on GPUs off-line. During the inference phase, the pre-trained network parameters can be loaded either from the cloud or from dedicated off-chip memory. Most recently, hardware accelerators for training have received widespread attention [42, 90, 79], but in this section we mainly focus on the inference phase in embedded settings.

Typically, an accelerator is composed of five parts: data buffers, parameter buffers, processing elements, global controller and off-chip transfer manager, as shown in Figure. 3 The data buffers are used to caching input images, intermediate data and output predictions, while the weight buffers are mainly used to cache convolutional filters. Processing elements are a collection of basic computing units that execute multiply-adds, non-linearity and any other functions such as normalization, quantization, etc. The global controller is used to orchestrate the computing flow on-chip, while off-chip transfers of data and instructions are conducted through a manager. This basic architecture can be found in existing accelerators designed for both specific and general tasks.

Heterogeneous computing is widely adopted in hard- 
ware acceleration. For computing-intensive operations such as multiply-adds, it is efficient to fit them on hardware for high throughout, otherwise, data pre-processing, softmax and any other graphic operations can be placed on the CPU/GPU for low latency processing.

\subsection{Processing Elements}

Among all of the accelerators, the biggest differences exist in the processing elements as they are designed for the majority of computing tasks in deep networks, such as massive multiply-add operations, normalization (batch normalization or local response normalization), and non-linearities (ReLU, sigmoid and tanh). Typically, the computing engine of an accelerator is composed of many small basic processing elements, as shown in Figure. 3, and this architecture is mainly designed for fully investing in data reuse and parallelism. However, there are many accelerators that operate with only one processing element in consideration of lower data movement and resource conservation [92, 57].

\subsection{Optimizing for High Throughput}

Since the majority of the computations in a network are matrix-matrix/matrix-vector multiplication, it is critical to deal with the massive nested loops to achieve high throughput. Loop optimization is one of the most frequently adopted techniques in accelerator design [92, 56, 73, 2, 88, 49], including loop tiling, loop unrolling, loop interchange, etc. Loop tiling is used to divide all of the data into multiple small blocks in order to alleviate the pressure of onchip storage [56, 2, 64], while loop unrolling attempts to improve the parallelism of the computing engine for high speed [56, 64]. Loop interchange determines the sequential computation order of the nested loops because different computation orders can result in significant differences in performance. The well-known systolic array can be seen as a combination of the loop optimization methods listed above, which leverage the nature of data locality and weight sharing in the network to achieve high throughput [37, 84].

SIMD-based computation is another way for high throughput. [60] presented a method for packing two lowbit multiplications into a single DSP block to double the computation, and [63] also proposed a SIMD-based architecture for speech recognition.

\subsection{Optimizing for Low Energy Consumption}

Existing works attempt to reduce the energy consumption of a hardware accelerator from both computing and I/O perspectives. [29] systematically illustrated the energy cost in terms of arithmetic operations and memory accesses. He demonstrated that operations based on integers are much more cheaper than their float-point counterparts, and lower bit integers are better. Therefore, most existing accelerators adopt low-bit or even binary data repre- sentation [98, 77, 61] to preserve energy efficiency. Most recently, logarithmic computation that transfers multiplications into bit shift operations has also shown its promise in energy savings [13, 16, 76].

Sparsity is gaining an increased popularity in accelerator design based on the observation that a great number of arithmetic operations can be discarded to obtain energy efficiency. [21], [20] and [62] designed architectures for image or speech recognition based on network pruning, while [1] and [95] proposed to eliminate ineffectual operations based on the inherent sparsity in networks.

Off-chip data transfers happen inordinately in hardware accelerators due to the fact that both network parameters and intermediate data are too large to fit on chip. [29] suggested that power consumption caused by DRAM access is several orders of magnitude of the SRAM, and therefore reducing off-chip transfers is a critical issue. [70] designed a flexible data buffing scheme to reduce bandwidth requirements, and [2] and [88] proposed a fusion-based method to reduce off-chip traffic. Most recently, [49] presented a block-based convolution that can completely avoid offchip transfers of intermediate data in VGG-16 with high throughput.

Many other approaches have been proposed to reduce power consumption. [94] used a pipelined FPGA cluster to realize acceleration, [7] presented an energy-efficient row stationary scheme to reduce data movements, and [102] attempted to reduce power consumption via low-rank approximation.

\subsection{Design Automation}

Recently, design automation frameworks that automatically map deep neural networks onto hardware are receiving wider attention. [83], [69], [78] and [84] proposed frameworks that automatically generate synthesizable accelerator for a given network. [55] presented an RTL compiler for FPGA implementation of diverse networks. [52] proposed an instruction set for hardware implementation, while [93] proposed a uniformed convolutional matrix multiplication representation for $\mathrm{CNNs}$.

\subsection{Emerging Techniques}

In the past few years, there have been many new techniques from both the algorithm side and the circuit side that have been adopted to implement fast and energy-efficient accelerators. Stochastic computing representing continuous values through streams of random bits have been investigated for hardware acceleration of deep neural networks [66, 71, 39]. On the hardware side, RRAM-based accelerators [5, 87] and the usage of 3-D DRAM [38, 14] have received greater attention. 


\section{Future Trends and Discussion}

In this section, we discuss some possible future directions in this field.

Non-fine-tuning or Unsupervised Compression. Most of the existing methods, including network pruning, lowrank compression and quantization, need labeled data to retrain the network for accuracy retention. The problems are twofold. First, labeled data is sometimes unavailable, as in medical images. Another problem is that retraining requires considerable human effort as well as professional knowledge. These two problems raise the need for unsupervised compression or even fine-tuning-free compression methods.

Scalable (Self-adaptive) Compression. Current compression methods have many hyperparameters that need to be determined ahead of time. For example, the sparsity of the network pruning, the rank of the decompositionbased methods or the bitwidth of fixed-point quantization methods. The selection of these hyperparameters is tedious work, which also requires professional experience. Thus, the investigation of methods that do not rely on humandesigned hyperparameters is a promising research topic. One direction may be to use annealing methods, or reinforcement learning.

Network Acceleration for Object Detection. Most of the model acceleration methods are optimized for image classification, yet very little effort has been devoted to the acceleration of other computer vision tasks such object detection. It seems that model acceleration methods for image classification can be directly used for detection. However, the deep neural networks for object detection or image segmentation are more sensitive to model acceleration methods, i.e., using the same model acceleration methods for object detection would suffer from a greater number of accuracy drops than with image classification. One reason for this phenomenon may be that object detection requires more complex feature representation than image classification. The design of model acceleration methods for object detection represents a challenge.

Hardware-software Co-design. To accelerate deep learning algorithms on dedicated hardware, a straightforward method is to pick up a model and design a corresponding architecture. However, the gap between algorithm modeling and hardware implementation will make it difficult to put this into practice. Recent advances in deep learning algorithms and hardware accelerators demonstrate that it is highly desirable to design hardware-efficient algorithms according to the low-level features of specific hardware platforms. This co-design methodology will be a trend in future work.

\section{Conclusion}

Deep neural networks provide impressive performance while suffering from huge computational complexity and high energy expenditure. In this paper, we provide a survey of recent advances in efficient processing of deep neural networks from both the algorithm and hardware points of view. In addition, we point out a few topics that deserve further investigation in the future.

\section{References}

[1] J. Albericio, P. Judd, T. Hetherington, T. Aamodt, N. E. Jerger, and A. Moshovos. Cnvlutin: Ineffectual-neuron-free deep neural network computing. In International Symposium on Computer Architecture, pages 1-13, 2016.

[2] M. Alwani, H. Chen, M. Ferdman, and P. A. Milder. Fusedlayer cnn accelerators. In MICRO, 2016.

[3] S. Anwar, K. Hwang, and W. Sung. Structured pruning of deep convolutional neural networks. ACM Journal on Emerging Technologies in Computing Systems (JETC), 13(3):32, 2017.

[4] Z. Cai, X. He, J. Sun, and N. Vasconcelos. Deep learning with low precision by half-wave gaussian quantization. July 2017.

[5] L. Chen, J. Li, Y. Chen, Q. Deng, J. Shen, X. Liang, and L. Jiang. Accelerator-friendly neural-network training: Learning variations and defects in rram crossbar. In Design, Automation and Test in Europe Conference and Exhibition, pages 19-24, 2017.

[6] Y. Chen, N. Sun, O. Temam, T. Luo, S. Liu, S. Zhang, L. He, J. Wang, L. Li, and T. Chen. Dadiannao: A machinelearning supercomputer. In Ieee/acm International Symposium on Microarchitecture, pages 609-622, 2014.

[7] Y. H. Chen, T. Krishna, J. S. Emer, and V. Sze. Eyeriss: An energy-efficient reconfigurable accelerator for deep convolutional neural networks. IEEE Journal of Solid-State Circuits, 52(1):127-138, 2017.

[8] J. Cheng, J. Wu, C. Leng, Y. Wang, and Q. Hu. Quantized cnn: A unified approach to accelerate and compress convolutional networks. IEEE Transactions on Neural Networks and Learning Systems (TNNLS), PP:1-14.

[9] Z. Cheng, D. Soudry, Z. Mao, and Z. Lan. Training binary multilayer neural networks for image classification using expectation backpropagation. arXiv preprint arXiv:1503.03562, 2015.

[10] M. Courbariaux, Y. Bengio, and J.-P. David. Binaryconnect: Training deep neural networks with binary weights during propagations. In Advances in Neural Information Processing Systems, pages 3123-3131, 2015.

[11] M. Denil, B. Shakibi, L. Dinh, N. de Freitas, et al. Predicting parameters in deep learning. In Advances in Neural Information Processing Systems, pages 2148-2156, 2013. 
[12] T. Dettmers. 8-bit approximations for parallelism in deep learning. arXiv preprint arXiv:1511.04561, 2015.

[13] Edward. Lognet: Energy-efficient neural networks using logarithmic computation. 2017 IEEE International Conference on Acoustics, Speech and Signal Processing (ICASSP), pages 5900-5904, 2017.

[14] M. Gao, J. Pu, X. Yang, M. Horowitz, and C. Kozyrakis. Tetris: Scalable and efficient neural network acceleration with 3d memory. In International Conference on Architectural Support for Programming Languages and Operating Systems, pages 751-764, 2017.

[15] Y. Gong, L. Liu, M. Yang, and L. Bourdev. Compressing deep convolutional networks using vector quantization. arXiv preprint arXiv:1412.6115, 2014.

[16] D. Gudovskiy and L. Rigazio. ShiftCNN: Generalized lowprecision architecture for inference of convolutional neural networks. arXiv preprint arXiv:1706.02393, 2017.

[17] Y. Guo, A. Yao, and Y. Chen. Dynamic network surgery for efficient dnns. In Advances In Neural Information Processing Systems, pages 1379-1387, 2016.

[18] S. Gupta, A. Agrawal, K. Gopalakrishnan, and P. Narayanan. Deep learning with limited numerical precision. In Proceedings of the 32nd International Conference on Machine Learning (ICML-15), pages 1737-1746, 2015.

[19] D. Hammerstrom. A vlsi architecture for highperformance, low-cost, on-chip learning. In IJCNN International Joint Conference on Neural Networks, pages 537544 vol.2, 2012.

[20] S. Han, J. Kang, H. Mao, Y. Hu, X. Li, Y. Li, D. Xie, H. Luo, S. Yao, and Y. Wang. Ese: Efficient speech recognition engine with sparse lstm on fpga. 2017.

[21] S. Han, X. Liu, H. Mao, J. Pu, A. Pedram, M. A. Horowitz, and W. J. Dally. Eie: Efficient inference engine on compressed deep neural network. In ACM/IEEE International Symposium on Computer Architecture, pages 243254, 2016.

[22] S. Han, H. Mao, and W. J. Dally. Deep compression: Compressing deep neural networks with pruning, trained quantization and huffman coding. arXiv preprint arXiv:1510.00149, 2015.

[23] S. Han, J. Pool, J. Tran, and W. Dally. Learning both weights and connections for efficient neural network. In Advances in Neural Information Processing Systems, pages 1135-1143, 2015.

[24] B. Hassibi and D. G. Stork. Second order derivatives for network pruning: Optimal brain surgeon. Morgan Kaufmann, 1993.

[25] K. He, X. Zhang, S. Ren, and J. Sun. Deep residual learning for image recognition. IEEE Conference on Computer Vision and Pattern Recognition (CVPR), 2016.

[26] Y. He, X. Zhang, and J. Sun. Channel pruning for accelerating very deep neural networks. arXiv preprint arXiv:1707.06168, 2017.

[27] G. Hinton, O. Vinyals, and J. Dean. Distilling the knowledge in a neural network. arXiv preprint arXiv:1503.02531, 2015.
[28] J. L. Holi and J. N. Hwang. Finite precision error analysis of neural network hardware implementations. In Ijcnn-91Seattle International Joint Conference on Neural Networks, pages 519-525 vol.1, 1993.

[29] M. Horowitz. 1.1 computing's energy problem (and what we can do about it). In Solid-State Circuits Conference Digest of Technical Papers, pages 10-14, 2014.

[30] L. Hou, Q. Yao, and J. T. Kwok. Loss-aware binarization of deep networks. arXiv preprint arXiv:1611.01600, 2016.

[31] A. G. Howard, M. Zhu, B. Chen, D. Kalenichenko, W. Wang, T. Weyand, M. Andreetto, and H. Adam. Mobilenets: Efficient convolutional neural networks for mobile vision applications. arXiv preprint arXiv:1704.04861, 2017.

[32] Q. Hu, P. Wang, and J. Cheng. From hashing to cnns: Training binary weight networks via hashing. In $A A A I$, February 2018.

[33] K. Hwang and W. Sung. Fixed-point feedforward deep neural network design using weights+ 1,0 , and- 1 . In 2014 IEEE Workshop on Signal Processing Systems (SiPS), pages 1-6. IEEE, 2014.

[34] F. N. Iandola, S. Han, M. W. Moskewicz, K. Ashraf, W. J. Dally, and K. Keutzer. Squeezenet: Alexnet-level accuracy with 50x fewer parameters and; $0.5 \mathrm{mb}$ model size. arXiv preprint arXiv:1602.07360, 2016.

[35] M. Jaderberg, A. Vedaldi, and A. Zisserman. Speeding up convolutional neural networks with low rank expansions. arXiv preprint arXiv: 1405.3866, 2014.

[36] H. Jegou, M. Douze, and C. Schmid. Product quantization for nearest neighbor search. IEEE transactions on pattern analysis and machine intelligence, 33(1):117-128, 2011.

[37] N. P. Jouppi. In-datacenter performance analysis of a tensor processing unit. In Proceedings of the 44th Annual International Symposium on Computer Architecture, ISCA '17, 2017.

[38] D. Kim, J. Kung, S. Chai, S. Yalamanchili, and S. Mukhopadhyay. Neurocube: A programmable digital neuromorphic architecture with high-density $3 \mathrm{~d}$ memory. In International Symposium on Computer Architecture, pages 380-392, 2016.

[39] K. Kim, J. Kim, J. Yu, J. Seo, J. Lee, and K. Choi. Dynamic energy-accuracy trade-off using stochastic computing in deep neural networks. In Design Automation Conference, page 124, 2016.

[40] M. Kim and P. Smaragdis. Bitwise neural networks. arXiv preprint arXiv:1601.06071, 2016.

[41] Y.-D. Kim, E. Park, S. Yoo, T. Choi, L. Yang, and D. Shin. Compression of deep convolutional neural networks for fast and low power mobile applications. arXiv preprint arXiv:1511.06530, 2015.

[42] J. H. Ko, B. Mudassar, T. Na, and S. Mukhopadhyay. Design of an energy-efficient accelerator for training of convolutional neural networks using frequency-domain computation. In Design Automation Conference, page 59, 2017.

[43] A. Krizhevsky and G. Hinton. Learning multiple layers of features from tiny images. 2009. 
[44] A. Krizhevsky, I. Sutskever, and G. E. Hinton. Imagenet classification with deep convolutional neural networks. In Advances in neural information processing systems, pages 1097-1105, 2012.

[45] V. Lebedev, Y. Ganin, M. Rakhuba, I. Oseledets, and V. Lempitsky. Speeding-up convolutional neural networks using fine-tuned $\mathrm{cp}$-decomposition. arXiv preprint arXiv:1412.6553, 2014.

[46] V. Lebedev and V. Lempitsky. Fast convnets using groupwise brain damage. In Proceedings of the IEEE Conference on Computer Vision and Pattern Recognition, pages 2554 2564, 2016.

[47] Y. LeCun, J. S. Denker, S. A. Solla, R. E. Howard, and L. D. Jackel. Optimal brain damage. In Advances in Neural Information Processing Systems, volume 89, 1989.

[48] F. Li, B. Zhang, and B. Liu. Ternary weight networks. arXiv preprint arXiv:1605.04711, 2016.

[49] G. Li, F. Li, T. Zhao, and J. Cheng. Block convolution: Towards memory-efficeint inference of large-scale cnns on fpga. In Design Automation and Test in Europe, 2018.

[50] M. Lin, Q. Chen, and S. Yan. Network in network. arXiv preprint arXiv:1312.4400, 2013.

[51] Z. Lin, M. Courbariaux, R. Memisevic, and Y. Bengio. Neural networks with few multiplications. arXiv preprint arXiv:1510.03009, 2015.

[52] S. Liu, Z. Du, J. Tao, D. Han, T. Luo, Y. Xie, Y. Chen, and T. Chen. Cambricon: An instruction set architecture for neural networks. SIGARCH Comput. Archit. News, 44(3), June 2016.

[53] Z. Liu, J. Li, Z. Shen, G. Huang, S. Yan, and C. Zhang. Learning efficient convolutional networks through network slimming. arxiv preprint, 1708, 2017.

[54] J.-H. Luo, J. Wu, and W. Lin. Thinet: A filter level pruning method for deep neural network compression. arXiv preprint arXiv:1707.06342, 2017.

[55] Y. Ma, Y. Cao, S. Vrudhula, and J. S. Seo. An automatic rtl compiler for high-throughput fpga implementation of diverse deep convolutional neural networks. In International Conference on Field Programmable Logic and Applications, pages 1-8, 2017.

[56] Y. Ma, Y. Cao, S. Vrudhula, and J.-s. Seo. Optimizing loop operation and dataflow in fpga acceleration of deep convolutional neural networks. In Proceedings of the 2017 ACM/SIGDA International Symposium on FieldProgrammable Gate Arrays, FPGA '17, 2017.

[57] Y. Ma, M. Kim, Y. Cao, S. Vrudhula, J. S. Seo, Y. Ma, M. Kim, Y. Cao, S. Vrudhula, and J. S. Seo. End-to-end scalable fpga accelerator for deep residual networks. In IEEE International Symposium on Circuits and Systems, pages 1-4, 2017.

[58] H. Mao, S. Han, J. Pool, W. Li, X. Liu, Y. Wang, and W. J. Dally. Exploring the regularity of sparse structure in convolutional neural networks. arXiv preprint arXiv:1705.08922, 2017.

[59] D. Miyashita, E. H. Lee, and B. Murmann. Convolutional neural networks using logarithmic data representation. arXiv preprint arXiv:1603.01025, 2016.
[60] D. Nguyen, D. Kim, and J. Lee. Double MAC: doubling the performance of convolutional neural networks on modern fpgas. In Design, Automation and Test in Europe Conference and Exhibition, DATE 2017, Lausanne, Switzerland, March 27-31, 2017, pages 890-893, 2017.

[61] Nurvitadhi. Can fpgas beat gpus in accelerating nextgeneration deep neural networks? In Proceedings of the 2017 ACM/SIGDA International Symposium on FieldProgrammable Gate Arrays, FPGA '17, 2017.

[62] A. Parashar, M. Rhu, A. Mukkara, A. Puglielli, R. Venkatesan, B. Khailany, J. Emer, S. W. Keckler, and W. J. Dally. Scnn: An accelerator for compressed-sparse convolutional neural networks. pages 27-40, 2017.

[63] M. Price, J. Glass, and A. P. Chandrakasan. 14.4 a scalable speech recognizer with deep-neural-network acoustic models and voice-activated power gating. In Solid-State Circuits Conference, pages 244-245, 2017.

[64] Qiu. Going deeper with embedded fpga platform for convolutional neural network. In Proceedings of the 2016 ACM/SIGDA International Symposium on FieldProgrammable Gate Arrays, FPGA '16, 2016.

[65] M. Rastegari, V. Ordonez, J. Redmon, and A. Farhadi. Xnor-net: Imagenet classification using binary convolutional neural networks. In ECCV (4), volume 9908, pages 525-542. Springer, 2016.

[66] A. Ren, Z. Li, C. Ding, Q. Qiu, Y. Wang, J. Li, X. Qian, and B. Yuan. Sc-denn: Highly-scalable deep convolutional neural network using stochastic computing. Acm Sigops Operating Systems Review, 51(2):405-418, 2017.

[67] A. Romero, N. Ballas, S. E. Kahou, A. Chassang, C. Gatta, and Y. Bengio. Fitnets: Hints for thin deep nets. arXiv preprint arXiv:1412.6550, 2014.

[68] O. Russakovsky, J. Deng, H. Su, J. Krause, S. Satheesh, S. Ma, Z. Huang, A. Karpathy, A. Khosla, M. Bernstein, et al. Imagenet large scale visual recognition challenge. International Journal of Computer Vision, 115(3):211-252, 2015.

[69] H. Sharma, J. Park, D. Mahajan, E. Amaro, J. K. Kim, C. Shao, A. Mishra, and H. Esmaeilzadeh. From highlevel deep neural models to fpgas. In Ieee/acm International Symposium on Microarchitecture, pages 1-12, 2016.

[70] Y. Shen, M. Ferdman, and P. Milder. Escher: A cnn accelerator with flexible buffering to minimize off-chip transfer. In IEEE International Symposium on Field-Programmable Custom Computing Machines, 2017.

[71] H. Sim and J. Lee. A new stochastic computing multiplier with application to deep convolutional neural networks. In Design Automation Conference, page 29, 2017.

[72] K. Simonyan and A. Zisserman. Very deep convolutional networks for large-scale image recognition. arXiv preprint arXiv:1409.1556, 2014.

[73] Suda. Throughput-optimized opencl-based fpga accelerator for large-scale convolutional neural networks. In Proceedings of the 2016 ACM/SIGDA International Symposium on Field-Programmable Gate Arrays, FPGA '16, 2016.

[74] C. Szegedy, W. Liu, Y. Jia, P. Sermanet, S. Reed, D. Anguelov, D. Erhan, V. Vanhoucke, and A. Rabinovich. 
Going deeper with convolutions. In Computer Vision and Pattern Recognition, pages 1-9, 2015.

[75] W. Tang, G. Hua, and L. Wang. How to train a compact binary neural network with high accuracy? In $A A A I$, pages 2625-2631, 2017.

[76] H. Tann, S. Hashemi, I. Bahar, and S. Reda. Hardwaresoftware codesign of accurate, multiplier-free deep neural networks. CoRR, abs/1705.04288, 2017.

[77] Umuroglu. Finn: A framework for fast, scalable binarized neural network inference. In Proceedings of the 2017 ACM/SIGDA International Symposium on FieldProgrammable Gate Arrays, FPGA '17, 2017.

[78] S. I. Venieris and C. S. Bouganis. fpgaconvnet: A framework for mapping convolutional neural networks on fpgas. In IEEE International Symposium on Field-Programmable Custom Computing Machines, pages 40-47, 2016.

[79] S. Venkataramani, A. Ranjan, S. Banerjee, D. Das, S. Avancha, A. Jagannathan, A. Durg, D. Nagaraj, B. Kaul, P. Dubey, and A. Raghunathan. Scaledeep: A scalable compute architecture for learning and evaluating deep networks. SIGARCH Comput. Archit. News, 45(2):13-26, June 2017.

[80] P. Wang and J. Cheng. Accelerating convolutional neural networks for mobile applications. In Proceedings of the 2016 ACM on Multimedia Conference, pages 541-545. ACM, 2016.

[81] P. Wang and J. Cheng. Fixed-point factorized networks. In The IEEE Conference on Computer Vision and Pattern Recognition (CVPR), July 2017.

[82] P. Wang, Q. Hu, Z. Fang, C. Zhao, and J. Cheng. Deepsearch: A fast image search framework for mobile devices. ACM Transactions on Multimedia Computing, Communications, and Applications (TOMM), 14, 2018.

[83] Y. Wang, J. Xu, Y. Han, H. Li, and X. Li. Deepburning: automatic generation of fpga-based learning accelerators for the neural network family. In Design Automation Conference, page 110, 2016.

[84] Wei. Automated systolic array architecture synthesis for high throughput cnn inference on fpgas. In Proceedings of the 54th Annual Design Automation Conference 2017, DAC '17, 2017.

[85] W. Wen, C. Wu, Y. Wang, Y. Chen, and H. Li. Learning structured sparsity in deep neural networks. In Advances in Neural Information Processing Systems, pages 2074-2082, 2016.

[86] J. Wu, C. Leng, Y. Wang, Q. Hu, and J. Cheng. Quantized convolutional neural networks for mobile devices. IEEE Conference on Computer Vision and Pattern Recognition (CVPR), 2016.

[87] L. Xia, T. Tang, W. Huangfu, M. Cheng, X. Yin, B. Li, Y. Wang, and H. Yang. Switched by input: Power efficient structure for rram-based convolutional neural network. In Design Automation Conference, page 125, 2016.

[88] Xiao. Exploring heterogeneous algorithms for accelerating deep convolutional neural networks on fpgas. In Proceedings of the 54th Annual Design Automation Conference 2017, DAC '17, 2017.
[89] S. Xie, R. Girshick, P. Dollar, Z. Tu, and K. He. Aggregated residual transformations for deep neural networks. In The IEEE Conference on Computer Vision and Pattern Recognition (CVPR), July 2017.

[90] H. Yang. Time: A training-in-memory architecture for memristor-based deep neural networks. In Design Automation Conference, page 26, 2017.

[91] S. Zagoruyko and N. Komodakis. Paying more attention to attention: Improving the performance of convolutional neural networks via attention transfer. arXiv preprint arXiv:1612.03928, 2016.

[92] Zhang. Optimizing fpga-based accelerator design for deep convolutional neural networks. In Proceedings of the 2015 ACM/SIGDA International Symposium on FieldProgrammable Gate Arrays, FPGA '15, 2015.

[93] C. Zhang, Z. Fang, P. Pan, P. Pan, and J. Cong. Caffeine: towards uniformed representation and acceleration for deep convolutional neural networks. In International Conference on Computer-Aided Design, page 12, 2016.

[94] C. Zhang, D. Wu, J. Sun, G. Sun, G. Luo, and J. Cong. Energy-efficient cnn implementation on a deeply pipelined fpga cluster. In Proceedings of the 2016 International Symposium on Low Power Electronics and Design, ISLPED '16, 2016.

[95] S. Zhang, Z. Du, L. Zhang, H. Lan, S. Liu, L. Li, Q. Guo, T. Chen, and Y. Chen. Cambricon-x: An accelerator for sparse neural networks. In Ieee/acm International Symposium on Microarchitecture, pages 1-12, 2016.

[96] X. Zhang, X. Zhou, M. Lin, and J. Sun. Shufflenet: An extremely efficient convolutional neural network for mobile devices. arXiv preprint arXiv:1707.01083, 2017.

[97] X. Zhang, J. Zou, K. He, and J. Sun. Accelerating very deep convolutional networks for classification and detection. IEEE Transactions on Pattern Analysis and Machine Intelligence (TPAMI), 2015.

[98] Zhao. Accelerating binarized convolutional neural networks with software-programmable fpgas. In Proceedings of the 2017 ACM/SIGDA International Symposium on Field-Programmable Gate Arrays, FPGA '17, 2017.

[99] A. Zhou, A. Yao, Y. Guo, L. Xu, and Y. Chen. Incremental network quantization: Towards lossless cnns with lowprecision weights. arXiv preprint arXiv:1702.03044, 2017.

[100] S. Zhou, Y. Wu, Z. Ni, X. Zhou, H. Wen, and Y. Zou. Dorefa-net: Training low bitwidth convolutional neural networks with low bitwidth gradients. arXiv preprint arXiv:1606.06160, 2016.

[101] C. Zhu, S. Han, H. Mao, and W. J. Dally. Trained ternary quantization. arXiv preprint arXiv: 1612.01064, 2016.

[102] J. Zhu, Z. Qian, and C. Y. Tsui. Lradnn: High-throughput and energy-efficient deep neural network accelerator using low rank approximation. In Asia and South Pacific Design Automation Conference, pages 581-586, 2016. 